
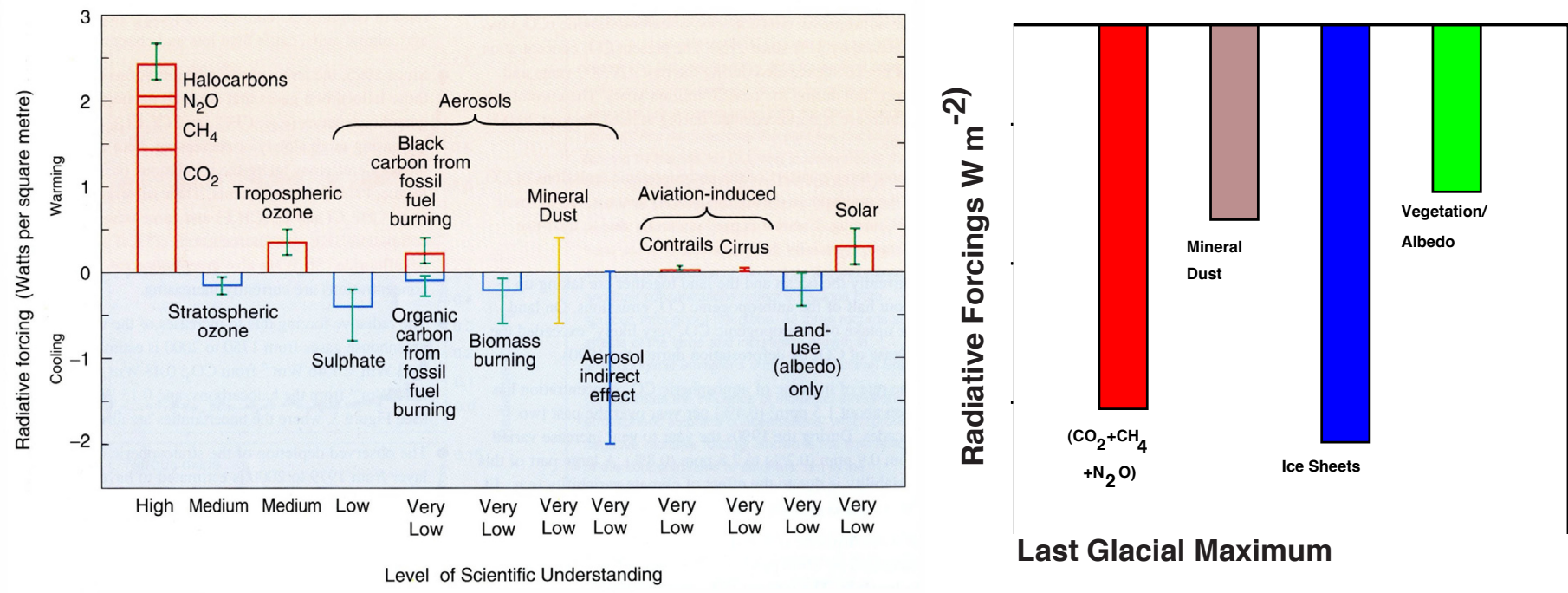

Figure 2: Radiative forcing for today (Ramaswamy et al., 2001, IPCC report 2001) (left) and the Last Glacial Maximum (right) relative to 1750 A.D. for the range of known perturbations.

of the Intergovernmental Panel on Climate Change, edited by Houghton, J. T., Y. Ding, D. J. Griggs, M. Noguer, P. J. van der Linden, X. Dai, et al., Cambridge University Press, 349-416, 2001.
Stauffer, B., J. Flückiger, E. Monnin, M. Schwander, J. M. Barnola, and J. Chappellaz, Atmospheric $\mathrm{CO}_{2}, \mathrm{CH}_{4}$ and $\mathrm{N}_{2} \mathrm{O}$ records over the past 60,000 years based on the comparison of different polar ice cores. Annals of Glaciology, 35, 202-208, 2002.

\title{
Solar forcing of climate change: Current status
}

\section{Judith Lean}

\section{E.0. Hulburt Center for Space Research, Naval Research Laboratory, Washington, DC, USA. jlean@ssd5.nrI.navy.mil}

\section{Solar Irradiance Variability}

\section{Observations}

Space-based radiometers have monitored the Sun's total irradiance since 1978 , providing a record that now covers more than two 11-year solar activity cycles. Composite irradiance time series have been constructed by combining individual measurements after first adjusting for instrumental differences. In the irradiance composite shown in Figure 1 (upper panel), levels are comparable (within $0.01 \%$ ) in the two most recent cycle minima, which suggests the absence of a secular trend in recent times (Fröhlich and Lean, 2004). This record agrees with models that parameterise irradiance variations in terms of sunspot and faculae, which respectively deplete and enhance local radiative output (Fröhlich and Lean, 2004).

Since 2003, instruments on SORCE (Solar Radiation and Climate Experiment) have been monitoring the Sun's total and spectral irradiance across ultraviolet, visible and near infrared regions, for the first time from space. On average, SORCE measures absolute total solar irradiances $5.2 \mathrm{Wm}^{-2}$ $(0.4 \%)$ lower than other radiometers. As expected, solar irradiance varies at all wavelengths in ways that reflect the different solar origins of the emissions from a range of temperatures and structures within the solar atmosphere. Maximum energy changes occur at wavelengths from 400 to $500 \mathrm{~nm}$, whereas fractional changes are greatest at UV wavelengths, where the energy change is, however, considerably smaller. A model that linearly combines sunspot and facular effects, incorporating the spectral dependence of their contrasts, provides close (but imperfect) tracking of both total and spectral irradiance variations (Lean et al., 2005).

\section{Reconstructions}

Until recently, reconstructions of historical solar irradiance assumed that longer-term variations are larger than during the 11-year cycle, since proxy indicators of solar activity (the aa index, ${ }^{14} \mathrm{C}$ and ${ }^{10} \mathrm{Be}$ cosmogenic isotopes in tree rings and ice cores, and the range of variability in Sun-like stars) suggest that the Sun is capable of a greater range of activity than witnessed in recent times. With this approach, total irradiance during the 17th century Maunder Minimum is reduced in the range of 0.15 to $0.4 \%$ ( 2 to $5 \mathrm{Wm}^{-2}$ ) below contemporary cycle minima values. But stellar data have been reassessed, instrumental drifts are suspected in the $a a$ index, and it has been shown that long-term trends in the $a a$ index and cosmogenic isotopes (generated by the open magnetic flux from the Sun that pervades the heliosphere) do not necessarily imply equivalent long-term trends in solar irradiance (which track closed magnetic flux within the solar atmosphere). In the irradiance reconstruction shown in Figure 1 (bottom panel), which is based on solar considerations alone (Wang, Lean and Sheeley, 2005), the amplitude of the background component is 0.27 times that of Lean (2000). As a result, total solar irradiance, increases about $\sim 0.5 \mathrm{Wm}^{-2}$ from the Maunder Minimum to the present-day quiet Sun. The larger amplitude secular irradiance changes of the initial reconstructions are therefore likely upper limits of long-term solar irradiance variability.

\section{Climate Response to Solar Variability \\ Mechanisms}

Distinctly different mechanisms are surmised for climate's response to solar radiative forcing. Direct "short-wave" heating of the surface, ocean and troposphere generates 
geographical and seasonal inhomogeneities that may alter land-atmosphere-ocean interactions. Climate may respond indirectly to stratospheric ozone changes driven by varying solar ultraviolet radiation. The altered altitudinal temperature gradient (from the troposphere to the stratosphere) and latitudinal gradient in the stratosphere (from the equator to the poles) couples the stratosphere to the troposphere radiatively and dynamically (Rind, 2002). A result of both direct and indirect solar forcing is thought to be the alteration of atmospheric circulation patterns, including the Hadley, Walker and Ferrel cells, with subsequent effects on, for example, rainfall patterns in tropical regions. A third mechanism involves modulation of the frequency and occurrence of internal modes of climate variability. Pacific sea surface temperature gradients arising from the deeper thermocline in the west Pacific Ocean relative to the east can affect ENSO. Solar UV irradiance changes may alter the high latitude stratosphere and the polar vortex, thereby affecting the NAO, which is observed to expand longitudinally to the Arctic annual oscillation during solar maxima. Furthermore, since the climate system exhibits significant "noise", the forcing may be amplified by stochastic resonance. Also possible are non-linear interactions of the forcing with existing cyclic modes.

\section{Empirical Evidence}

A linear combination of solar, anthropogenic, volcanic and ENSO influences accounts for approximately $50 \%$ of the observed variance in global surface temperature between 1979 and 2004, based on multivariate linear regression. As Figure 2 (left panel) shows, the surface warms $0.1^{\circ} \mathrm{C}$ at solar cycle maxima (forcing of $0.2 \mathrm{Wm}^{-2}$ ) and $0.39^{\circ} \mathrm{C}$ overall from anthropogenic influences (forcing of $1 \mathrm{Wm}-2$ ). ENSO and volcanic activity produce episodic fluctuations that can exceed $0.2^{\circ} \mathrm{C}$. A $0.1^{\circ} \mathrm{C}$ surface temperature response to solar forcing approximately in-phase with the solar cycle is inconsistent with current understanding that oceanic thermal inertia strongly dampens (by a factor of five) decadal forcing. Thus, the effect likely involves the atmosphere and surface but does not engage the deep ocean. Consistent with this, the solar signal strength grows with altitude in the Earth's atmosphere, to $0.3^{\circ} \mathrm{C}$ at $10 \mathrm{~km}$ and $1^{\circ} \mathrm{C}$ at $50 \mathrm{~km}$. The right panel of Figure 2 indicates solar cycle changes of $0.4^{\circ} \mathrm{C}$ in lower stratospheric temperatures measured by the Microwave Sounding Unit. At these altitudes, the variations track the solar UV irradiance, ENSO effects are minimal $\left(<0.05^{\circ} \mathrm{C}\right)$ and volcanic influences can exceed $1^{\circ} \mathrm{C}$.

Figure 1. Shown in the top panel is the observational record of total solar irradiance of Fröhlich and Lean (2004). In the bottom panel, the upper envelope of the shaded region is total . solar irradiance variations arising from the 11-year activity cycle. The lower envelope is the total irradiance reconstructed by Lean (2000), in which the long-term trend was inferred from brightness changes in Sun-like stars. In comparison is the recent reconstruction of Wang, Lean and Sheeley (2005), using a flux transport model to simulate the long-term evolution of the closed flux that generates bright faculae.
The relative influences of solar and other climate forcings are less certain prior to the era of space-based observations. Solar-related global warming since the 17th century Maunder Minimum is likely of order $0.1^{\circ} \mathrm{C}$, or less, which is smaller than suggested by previous studies in which reconstructed solar irradiance changes were larger. On longer timescales, coherent variations in high-resolution paleo-records at quite different geographical locations in phase with solar activity (as indicated by the ${ }^{14} \mathrm{C}$ and ${ }^{10} \mathrm{Be}$ cosmogenic isotopes) suggest a global response to solar forcing. Drought and rainfall seem particularly sensitive to solar variability, especially in vulnerable geographical regions such as those in the vicinity of the ITCZ. Crucial for interpreting paleo Sun-climate relationships is improved understanding of ${ }^{14} \mathrm{C}$ and ${ }^{10} \mathrm{Be}$ modulation processes. The isotope records actually reflect solar-induced changes that impede the passage of cosmic rays through the plasma environment near the Earth, not the closed magnetic fields in sunspots and faculae that modulate irradiance. Since climate itself can affect the deposition of the cosmogenic isotopes in ice and trees, the cosmogenic records may also indicate ocean (rather than solar) variability. Ultimately, a complete specification of the physical processes within the entire Sun-Earth system is needed to resolve these issues.

\section{Acknowledgments}

NASA funded this work. Ongoing collaborations with Claus Fröhlich, David Rind and Yi-Ming Wang are gratefully acknowledged. The text is adapted from Lean et al. (2005).

\section{References}

Fröhlich, C. and Lean, J.: 2004, Solar radiative output and its variability: evidence and mechanisms, Astron. Astrophys. Rev. 12, 4, 273-320.

Lean, J.: 2000, Evolution of the Sun's Spectral Irradiance since the Maunder Minimum, Geophys. Res. Lett. 27, 2425-2428.

Total Solar Irradiance
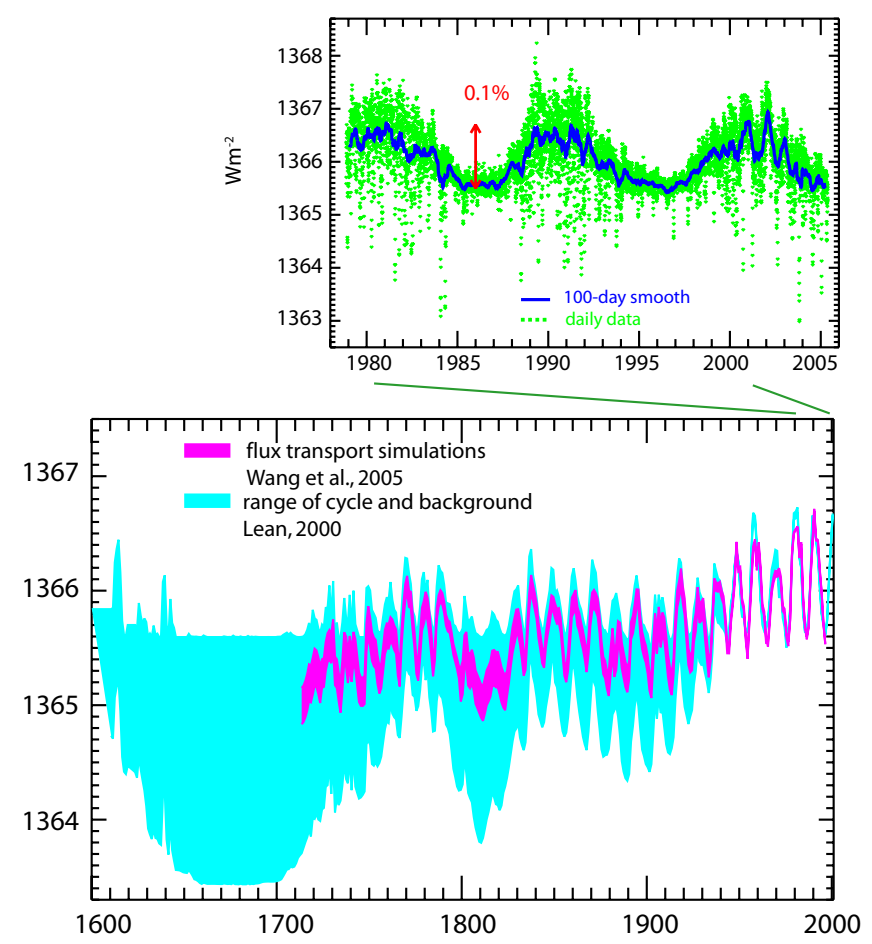
Figure 2. Comparison of different sources of variance in Earth's recent surface (left) and lower stratospheric (right) temperatures, deduced from multivariate linear regression. The volcanic aerosols and ENSO indices are lagged by 6 months, and solar irradiance by one month, relative to the observed surface temperatures, but in the lower stratosphere all lags are zero.
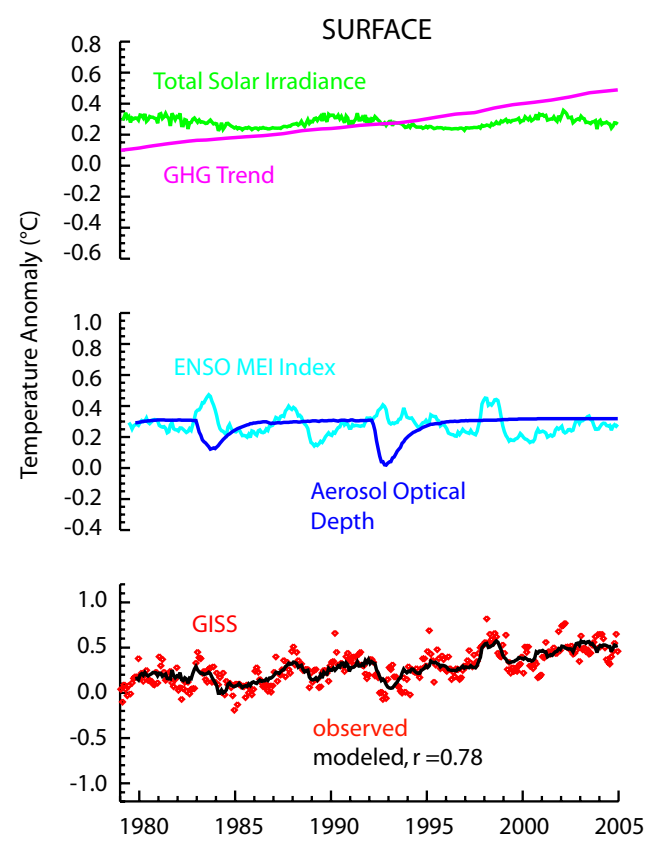

LOWER STRATOSPHERE
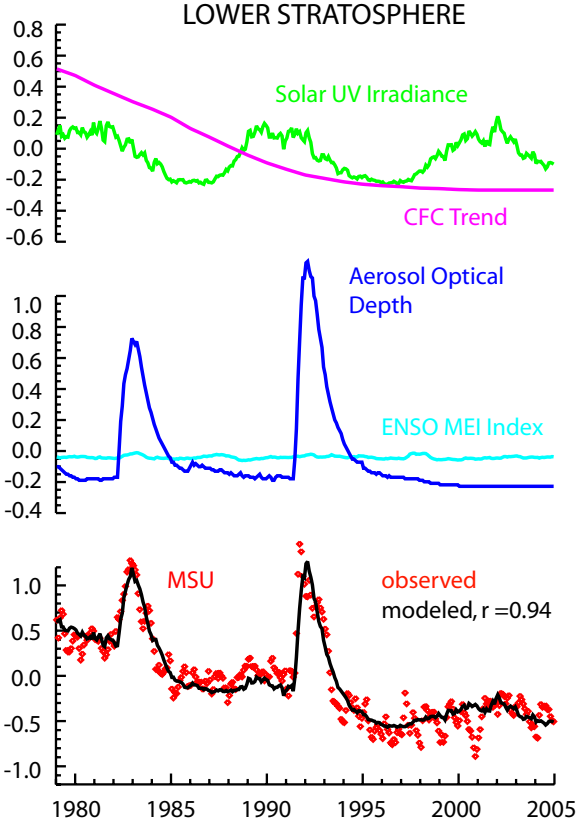

Lean, J., Rottman, G., Harder, J., and Kopp, G.: 2005, SORCE contributions to new understanding of global change and solar variability, Solar Phys., in press.

Rind, D.: 2002, The Sun's role in climate variations, Science
Wang, Y-. M., Lean, J. L., and Sheeley, Jr., N. R.: 2005, Modeling the Sun's magnetic field and irradiance since 1713, Astrophys. J. 625, 522-538.

296, 673-677.

\section{Aerosol effects on clouds and climate}

\section{Ulrike Lohmann}

\section{Institute for Atmospheric and Climate Science, ETH Zürich, Zurich, Switzerland; ulrike.Iohmann@env.ethz.ch}

The burning of fossil fuels and biofuels due to human activities has greatly increased the amount of particulate matter in the atmosphere. The major aerosol components are mineral dust, sea salt, sulfates, nitrates, black carbon (also termed soot) and particulate organic matter (POM). The natural aerosol species, mineral dust and sea salt, dominate the mass concentration in the atmosphere. On average, they contribute $39 \mathrm{mg} / \mathrm{m}^{2}$ and $13 \mathrm{mg} / \mathrm{m}^{2}$, respectively, whereas the anthropogenic components, sulfate, POM and black carbon only contribute 3.9, 3.3 and $0.4 \mathrm{mg} / \mathrm{m}^{2}$, respectively, to the annual global average as deduced from 20 different global models (Kinne et al., 2005). So far, nitrate is not included in most models because of its semi-volatile nature.

Optically, mineral dust and sea salt are less important because of their larger size. Thus, they each contribute only as much to the aerosol optical depth as sulfate does $(25 \%)$. Black carbon, which contributes only $3 \%$ to the optical depth, is the main aerosol type that absorbs solar radiation and can lead to a warming of the surrounding air. This warming can prevent cloud formation because the atmosphere becomes more stable, or even lead to an evaporation of cloud droplets. This semi-direct effect thus counteracts some of the negative aerosol forcings from scattering aerosols, such as sea salt and sulfate, at the top of the atmosphere (e.g., Lohmann and Feichter, 2005).

At the Earth's surface, however, both scattering and absorbing aerosols work in the same direction to reduce the amount of solar radiation reaching the surface. Since pre-industrial times, increasing emissions of aerosols as a result of human activity have caused a reduction of solar radiation at the surface ("solar dimming") by increasing aerosol and cloud optical depth. Such a reduction of $1.3 \%$ per decade over the land surfaces from 1961 to 1990 has been observed in many regions worldwide (e.g., Wild et al., 2004). Recent surface observations, however, show that the long term decline in solar radiation at land surfaces turned into an increase in surface solar radiation during the 1980s (Wild et al., 2005), in agreement with recent emission trends in the "old" industrial regions in the northern hemisphere (Krüger and Graßl, 2002), as well as with long-term black carbon trends in the Canadian Arctic (Sharma et al., 2004), and sulfate deposition declines over Europe and North America since 1978 (E. Holland, pers. comm.).

Aerosols also act as centres for cloud droplets and ice crystals, thereby changing cloud properties. If more aerosols compete for the uptake of water vapour, the resulting cloud droplets do not grow as large. More smaller cloud droplets have a larger surface area than fewer larger cloud droplets for the same amount of cloud water. Thus, a polluted cloud reflects more solar radiation back to space, resulting in a negative radiative forcing at TOA (cloud albedo effect). In addition, these more numerous but smaller cloud droplets collide less efficiently with each other, which reduces the precipitation efficiency of polluted clouds and prolongs their lifetime. It also implies more scattering of solar radiation back into space, thus reinforcing the cloud albedo effect. Whether the cloud lifetime or the cloud albedo effect is more important is still an open question. Whereas some models predict that the cloud albedo effect is four times as 